Disponível em

http://www.anpad.org.br/rac

RAC, Rio de Janeiro, v. 16, n. 5, art. 1, pp. 646-663, Set./Out. 2012

$(\mathrm{coc}$ EY-NG

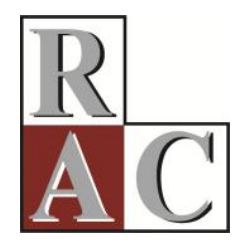

\title{
Antecedentes da Adoção da Computação em Nuvem: Efeitos da Infraestrutura, Investimento e Porte
}

Antecedents of the Adoption of Cloud Computing: Effects of Infrastructure, Investment and Size

Otavio Prospero Sanchez *

E-mail: otavio.sanchez@ fgv.br Fundação Getúlio Vargas - EAESP/FGV

São Paulo, SP, Brasil.

Alexandre Cappellozza

E-mail: cappellozza@gmail.com Fundação Getúlio Vargas - EAESP/FGV

São Paulo, SP, Brasil.

\footnotetext{
* Endereço: Otavio Prospero Sanchez

Rua Itapeva, 474, $9^{\circ}$ andar, São Paulo/SP, 01332-000.
} 


\title{
Resumo
}

A computação em nuvem, uma forma peculiar de armazenamento e disponibilização de informações, tem despertado muito interesse pelo seu potencial de alterar significativamente os investimentos em infraestrutura de Tecnologia da Informação (TI). Paradoxalmente, ao mesmo tempo em que surge o benefício para as organizações da possibilidade de executarem computação em nuvem, os decisores deparam-se com o dilema advindo da possibilidade de que concorrentes tenham acesso a recursos anteriormente escassos ou diferenciais, fontes de vantagem competitiva. A questão que emerge é saber se a computação em nuvem aumenta a complexidade da decisão de investimentos ou, em oposição, simplifica essa análise, ao permitir que recursos sejam liberados para investimentos em outras competências requeridas pelo negócio. Esta pesquisa investiga quais são os antecedentes associados à decisão de efetuar computação em nuvem e avalia os efeitos da infraestrutura de TI existente, do porte e do montante de investimentos nessa decisão. Por meio da análise de dados secundários, apresenta um modelo estrutural acompanhado de explicações sobre as relações encontradas. As contribuições do artigo envolvem a explicação teórica sobre o problema da decisão de investimentos em TI nesse contexto e da decisão por adotar computação em nuvem, adicionalmente, apresenta algumas implicações gerenciais desses achados. Limitações da pesquisa e sugestões para evoluções em estudos futuros são apresentadas.

Palavras-chave: infraestrutura de TI; computação em nuvem; investimentos em TI.

\begin{abstract}
Cloud computing, a peculiar form of storage and of making information available, has attracted much interest for its potential to significantly alter required investment in information technology infrastructure. As a paradox, while organizations can benefit from the possibility of implementing cloud computing, decision makers are faced with the dilemma arising from the possibility that competitors will have access to previously scarce or unique resources, essentially sources of competitive advantage. The question that arises is whether cloud computing increases the complexity of investment decisions or, in contrast, simplifies the analysis by allowing resources to be released for investment in other skills required by the business. This research investigates what antecedents are associated with the decision to undertake cloud computing and evaluates the effects existing IT infrastructure and the size and amount of investments have in this decision. Analysis of secondary data was used to develop a structural model, which is accompanied by explanations of the relationships uncovered. This paper's contributions include a theoretical explanation of the quandary related to IT investments within this context and the decision to adopt cloud computing and, additionally, the presentation of some managerial implications of these findings. Research limitations and suggestions for changes in future studies are also presented.
\end{abstract}

Key words: IT infrastructure; cloud computing; IT investments. 


\section{Introdução}

Grande parte da literatura de investimentos em tecnologia da informação (TI) descreve sua importância estratégica na obtenção e manutenção de vantagens competitivas para as organizações. $\mathrm{O}$ entendimento de que a tecnologia da informação é um recurso importante para permitir executar os processos, baixar custos e controlar atividades é bastante disseminado.

Entretanto, do ponto de vista das teorias de estratégia, especialmente da teoria baseada em recursos (RBV), essa vantagem competitiva não é sustentável se o recurso for facilmente imitável ou estiver acessível aos competidores. Em vista disso, a decisão de investir ou contratar tecnologia da informação envolve diversos dilemas que obrigam o gestor a ponderar sobre sua capacidade de investimento, seus objetivos estratégicos e quanto aos movimentos de seus competidores. Especialmente porque a tecnologia da informação é um recurso caro e perecível, a decisão sobre como investir assume grande relevância (Sanchez \& Albertin, 2008).

Como um novo cenário, os recentes avanços na disponibilização de soluções de computação em nuvem vêm sendo vistos como uma nova possibilidade para a redução nos volumes de investimento em tecnologia da informação, habilitando maior flexibilidade na demanda por serviços, com custos bastante atrativos (Bublitz, 2010; Ford, 2010). Organizações poderiam reter seus recursos financeiros para aumentar sua capacidade de investimento em atividades fundamentais de seu negócio, deixando para fornecedores a administração dos ativos de tecnologia.

Um desafio, nesse novo cenário, é avaliar se usar as capacidades disponibilizadas por fornecedores poderia levar à redução dos diferenciais competitivos das organizações, dessa maneira, tornando-se uma ameaça às suas posições competitivas. Ao mesmo tempo, competidores podem adquirir vantagens se tiverem acesso a ofertas de fornecedores que repassem ganhos derivados das significativas economias de escala obtidas na execução dos processos de tecnologia (Brynjolfsson \& Hitt, 2000).

Esta pesquisa explora a necessidade de identificação dos fatores antecedentes da adoção de computação em nuvem por parte das organizações. Ao identificar os mecanismos de escolha das organizações, contribui para o entendimento do problema e para a prática gerencial, que pode beneficiar-se dos resultados de melhores tomadas de decisão a respeito da adoção de computação em nuvem.

A estrutura do artigo $^{(1)}$ consiste da revisão de literatura sobre infraestrutura de tecnologia da informação, economias de escala e decisões de investimento e a respeito da própria computação em nuvem, desenvolvendo argumentos atinentes a possíveis relações apresentadas em forma de enunciados de hipóteses e modelo de relações. Em seguida, apresenta a operacionalização da pesquisa para analisar a validade estrutural do modelo, coloca os resultados, discute os achados e expõe conclusões, bem como as limitações e propostas para evoluções futuras.

\section{Revisão de Literatura}

\section{Infraestrutura de tecnologia da informação}

O conceito de infraestrutura de tecnologia da informação pode ser definido como um conjunto de recursos tecnológicos que suportam a operacionalização de negócios correntes ou futuros (Byrd \& Turner, 2000). Na visão tradicional, a infraestrutura de tecnologia da informação consiste de recursos centralizados de informação que podem compreender hardware e software, dispositivos de telecomunicações e redes (Armstrong \& Sambamurthy, 1999; Byrd, Pitts, Adrian, \& Davidson, 2008). Outra visão focaliza a habilidade de exercer as funcionalidades dos sistemas de informação entre 
departamentos da empresa e múltiplas unidades de negócio. Por este conceito, profissionais, conhecimento e expertise, políticas, além de serviços compartilhados de TI e bases de dados, são recursos fundamentais compreendidos no conceito de infraestrutura de TI (Duncan, 1995; Renkema, 1998). Além dos equipamentos e sistemas, a infraestrutura de TI de uma organização também pode ser composta de profissionais da área e conhecimentos, entre outros componentes (Weill \& Broadbent, 1998).

No tocante aos componentes da infraestrutura de TI, McAfee (2006) apresenta três categorias:

1. Funcionais: compreende sistemas que auxiliam os usuários em tarefas discretas, tais como: softwares processadores de textos e planilhas, entre outros.

2. Rede: referem-se a softwares que possibilitam a interação entre os interessados, por exemplo: gerenciadores de e-mail, mensagens instantâneas ou outros sistemas.

3. Corporativo: inclui os sistemas desenvolvidos para gerenciamento e execução de processos específicos da organização, tais como os sistemas integrados Enterprise Resource Planning (ERP).

Outra definição de infraestrutura de TI está associada à capacidade de prover conectividade e flexibilidade à tecnologia da organização. Nesse contexto, uma infraestrutura flexível é aquela que supre demandas de compatibilidade, modularidade e conectividade com outros componentes tecnológicos (Duncan, 1995). Assim, uma infraestrutura caracterizada como flexível poderia absorver uma ampla variedade de hardwares, softwares, dados, plataformas de comunicação e suportar a conexão entre a camada física de dispositivos com as mais variadas demandas humanas intrínsecas às atividades da organização. Vale notar que esta conexão entre as diversas interfaces da infraestrutura de TI (máquina e humana) pode ainda estar em locais geograficamente distintos (Byrd \& Turner, 2000; Duncan, 1995). Em outras palavras, entende-se que se pode entender infraestrutura de TI como os meios pelos quais se pode proporcionar acesso pleno aos sistemas e dados da organização, o que pode conceder aos usuários mobilidade, acesso ubíquo e compartilhamento de informações (Duncan, 1995; Simon, 1990).

\section{Economias de escala e decisões de investimentos em TI}

A literatura de investimentos em tecnologia da informação, em grande parte, reconhece o caráter estratégico que esse recurso pode ter na obtenção e sustentação de vantagens competitivas. A ideia de que a tecnologia da informação apoia operações e que as organizações devem investir na aquisição e domínio desse recurso encontra pouca resistência (Sanchez \& Albertin, 2009). A literatura ainda sugere que os estrategistas da organização tenham parcimônia na decisão de investimentos em TI, uma vez que parte desses recursos pode ser copiada pelos concorrentes, o que pode minar a vantagem competitiva obtida pela aquisição de tecnologia em razão dessa adoção posterior de tais recursos pelos concorrentes. Por sua vez, resistir a investir em TI pode deixar a organização em posição vulnerável em seu mercado, em comparação com seus concorrentes (Clemons \& Weber, 1990).

A velocidade com que ocorrem os avanços de variados tipos de tecnologia da informação é um aspecto ainda mais divulgado, e o argumento de que, a cada dia, a sociedade é capaz de contar com novas, melhores e mais baratas tecnologias, também, é corriqueiro. Menor atenção, entretanto, verifica-se no argumento de que a velocidade de avanço é acompanhada por uma possivelmente igual ou até maior velocidade de obsolescência de tecnologias que podem se tornar um grave problema de desinvestimento em organizações, pois a taxa em que ocorre a necessidade de reinvestimento ainda é usualmente muito superior em TI do que em outros tipos de ativos (Brynjolfsson \& Hitt, 2000).

Quando se trata de tecnologias de infraestrutura, o problema de desinvestimento pode tornar-se muito sério à medida em que essas tecnologias exercem um papel de plataforma na qual se apoiam outras tecnologias e sistemas que conduzem todos os negócios. É de esperar, portanto, que os decisores prefiram tecnologias de infraestrutura mais estáveis e flexíveis, de maneira a reduzir o risco 
de desinvestimento por falta de reposição de funcionalidades perdidas em função da obsolescência tecnológica.

A relativa maior estabilidade desse tipo de tecnologia em relação a outras, em combinação com a relativa maior informatização que as empresas experimentam em seus ambientes de negócio dia após dia (Meirelles, 2011), torna possível o surgimento de economias de escala. Essas economias de escala configuram-se como padronizações de sistemas que acabam por adquirir volumes capazes de permitir sua administração de maneira mais produtiva.

A infraestrutura de TI apresenta, então, duas características as quais tornam esse tipo de tecnologia adequado para que as organizações considerem na hora de investir: ciclos de renovação mais lentos que outros tipos de tecnologia e potencial de economias de escala. Por sua vez, a obsolescência da TI não deixa de ser um problema presente, mesmo considerando que a infraestrutura tenha um ciclo de renovação de padrões tecnológicos mais lento que as aplicações. Argumentamos que a combinação desses efeitos leva organizações com altos níveis de infraestrutura a necessitarem de volumes proporcionalmente maiores de investimento para a adequada reposição de suas capacidades de operação, ainda que possam existir economias de escala na manutenção desses recursos de tecnologia da informação. Com base nessa premissa, declaramos a proposição da Hipótese 1:

H1: No contexto das organizações, incrementos na infraestrutura de TI estão positivamente associados a incrementos de investimentos em TI.

Ainda que as tecnologias de infraestrutura ofereçam padrões tecnológicos relativamente mais estáveis que outros tipos de tecnologia da informação, como, por exemplo, aplicações, de uma forma geral, a tecnologia da informação envolve riscos e conhecimento técnico específico. A velocidade de surgimento de novos padrões no campo da tecnologia da informação é uma característica que torna as decisões sobre investimento mais complexas em razão do potencial de rápida obsolescência dos ativos. Essa característica faz com que decisores usualmente não sejam hábeis em tomar decisões plenamente racionais no tocante a investimentos desse tipo. Diversos aspectos podem influenciar suas decisões, tais como ancoragem em experiências anteriores, crenças, informações incompletas, pouca racionalidade, entre outros (Bannister \& Remenyi, 2000; Clemons \& Weber, 1990; Holm \& Rikhardsson, 2008; Renkema, 1998; Sanchez \& Albertin, 2009). Esses aspectos podem trazer grandes dificuldades para os gestores avaliarem adequadamente os aportes de recursos sobre a infraestrutura necessária para operacionalização dos sistemas de informação, uma vez que estes aportes podem gerar benefícios indiretos e intangíveis (Clemons \& Weber, 1990; Davern \& Kauffman, 2000; Ryan \& Harrison, 2000).

Os custos de coordenação observados nas organizações, por sua vez, são crescentes à medida que o seu porte aumenta, o que demanda a coordenação necessária para fazer frente à complexidade existente em expressivas coleções de processos típicas de grandes operações (Sanchez \& Albertin, 2009). A solução tradicional para esse problema é fazer com que a infraestrutura de tecnologia acompanhe o porte da organização, pois um dos principais argumentos a favor de efetuar investimentos de infraestrutura de TI é a possibilidade de aumento da eficiência das operações conjugado com a redução de custos operacionais, por meio da redução de custos de transação e coordenação (Duncan, 1995; Renkema, 1998).

Mesmo que não se tenha uma clara justificativa para os investimentos feitos em tecnologia (Santos, 1991), estudos na área de tecnologia indicam que os investimentos em TI efetuados pelas organizações usualmente se processam como taxas crescentes do faturamento ao longo do tempo (Meirelles, 2011; Sanchez \& Albertin, 2009).

Considerando a clássica associação entre porte e capacidade de competição, pode-se argumentar que organizações de maior porte, ao adquirem a liderança em seus mercados, tornam-se paradigmas de processos e desempenho. Estar nessa posição usualmente estimula o surgimento de novos competidores menores em múltiplas frentes simultaneamente, expondo a organização líder em cada segmento em que atua (Damanpour, 2010). Em consequência, organizações de maior porte tendem a 
ser pressionadas por múltiplos agentes que podem se especializar em segmentos menores e que são potencialmente mais ágeis às demandas desses segmentos. Uma resposta estratégica natural de organizações de maior porte é enfatizar os ganhos de produtividade advindos de seu tamanho, aproveitando as economias de escala produzidas como forma de reduzir sua vulnerabilidade e compensar eventual diferença de agilidade (Gomes, Kruglianskas, \& Scherer, 2009).

Assim, conjecturamos que as condições de competitividade às quais organizações de maior porte estão submetidas tendem a exercer pressões crescentes por investimentos em ativos de infraestrutura de TI. Adicionalmente, a pressão pela redução dos custos de coordenação crescentes com o porte, adicionada aos argumentos de produtividade, leva a formular a Hipótese 2:

H2: Incrementos no porte da organização estão positivamente associados a incrementos de investimentos em TI.

\section{Computação em nuvem}

O termo computação em nuvem é sucessor de outro conceito na área de tecnologia, o de virtualização digital. Uma definição para a virtualização consiste da execução de softwares em servidores, com disponibilidade de acesso externo (Fano \& Gershman, 2002). Já o conceito de computação em nuvem é definido como a disponibilidade de aplicações computacionais oferecidas como serviços a partir de acesso via Internet, por meio de hardware e software hospedados em datacenters remotos (Armbrust et al., 2010).

Dentre as características principais dos serviços ofertados como computação em nuvem, pode-se citar o acesso sob demanda: o atendimento da demanda de recursos computacionais à medida do requerido pelo processo, o que faz com que o acréscimo ou redução de recursos computacionais seja orientado pelo cliente, geralmente, conforme suas necessidades; o pay-per-use: que consiste de calcular os pagamentos aos fornecedores de acordo com a utilização dos serviços; a conectividade: característica que requer que o acesso aos servidores seja realizado em alta velocidade e que permite o tráfego de informações em grande volume; o compartilhamento: que se refere à possibilidade de obtenção de ganhos de escala nas receitas dos serviços de computação em nuvem pelo compartilhamento do excesso de capacidade de infraestrutura de TI entre grupos de clientes; a abstração: cujos clientes podem desconhecer completamente o local físico de hospedagem de suas informações, uma vez que a segurança de disponibilidade é definida por meio da especificação prévia de níveis de serviço a serem atendidos pelo fornecedor; o comprometimento: que incluem cláusulas específicas a respeito de garantias de nível de serviço a respeito de condições de atendimento de demandas que podem ser desfavoráveis aos clientes (Durkee, 2010; Katzan, 2010).

Fornecedores de serviços de computação em nuvem podem ofertar serviços em diferentes níveis de abstração, conforme segue (Iyer \& Henderson, 2010; Katzan, 2010; Ryan \& Loeffler, 2010):

Infraestrutura - Cloud Infrastructure as a Service - IaaS: são oferecidos servidores dedicados para acesso e controle total do cliente que pode armazenar, manipular suas informações e proceder a instalação de aplicativos e sistemas operacionais de acordo com suas necessidades;

Plataforma - Cloud Platform as a Service - PaaS: neste caso, os fornecedores concedem ambientes virtuais, incluindo sistemas e linguagens de programação, propícios para o desenvolvimento de aplicações pelos clientes. Sendo assim, tais clientes não precisam se preocupar com outros processos inerentes às operações dos servidores;

Software-Cloud Software as a Service - SaaS: o fornecedor concede os sistemas e aplicações, em ambientes virtuais, com interfaces amigáveis destinadas à utilização de usuários comuns. Como exemplo deste tipo de oferta, pode ser citado o serviço de e-mail acessado pelo navegador, também, denominado webmail. 
O serviço de computação em nuvem pode ainda ser classificado quanto à restrição de acesso aos usuários, nas seguintes categorias (Katzan, 2010; Ryan \& Loeffler, 2010):

Nuvem Pública: a infraestrutura da nuvem é disponibilizada para o público em geral.

Nuvem Privada: o gerenciamento e operação da nuvem são realizados por uma organização e o acesso às informações pode ser restrito por políticas de segurança.

- Nuvem Comunitária: neste caso, a infraestrutura da nuvem é administrada por um conjunto de organizações e cujo gerenciamento pode estar sujeito a regras estabelecidas pela comunidade proprietária.

Nuvem Híbrida: trata-se de um grupo de nuvens, embora estas nuvens mantenham sua identidade diferenciada entre o grupo, podem ser do tipo privada, pública ou comunitária. As nuvens pertencentes a esta categoria podem estar associadas entre si por protocolos ou padrões técnicos.

Os benefícios que podem ser obtidos pelas empresas pela utilização da computação em nuvem incluem a redução de custos associados ao consumo de energia elétrica dos clientes, a agilidade para expansão ou redução de recursos computacionais na medida das necessidades de negócio, a diminuição dos custos operacionais da TI, a disponibilização de tempo às equipes internas de TI para outras atividades e o atendimento a eventuais picos de demandas de recursos tecnológicos (Fano \& Gershman, 2002).

Por sua vez, organizações que sejam capazes de gerar economias de escala em suas atividades, via de regra como resultado da produtividade associada ao seu tamanho, podem tornar-se atrativas para adquirir as melhores práticas e recursos de mercado. Com isso, podem conseguir acesso aos melhores talentos, recursos e técnicas disponíveis no mercado (Barua, Kriebel, \& Mukhopadhyay, 1991). Devido à restrição da capacidade de geração de recursos livres para investimento, a adoção de computação em nuvem pode ser vista como uma forma de liberação de recursos, a qual faria mais sentido para empresas que necessitam adquirir posição competitiva do que para as organizações que já a possuem. Então, organizações não líderes seriam mais propensas a adotarem novas práticas que liberem recursos para investimento em suas competências centrais de negócio, enquanto que as organizações de maior porte, estando em melhor posição para adquirirem economias de escala, tenderiam a ser mais refratárias à adoção de novas práticas que mudem as regras estabelecidas da competição. Em vista desses aspectos, enunciamos a seguinte Hipótese para o estudo:

H3: Incrementos no porte das organizações estão negativamente associados a incrementos na possibilidade de adoção da computação em nuvem.

Por fim, pesquisas indicam que a TI é um recurso caro e que tende a compor ao longo do tempo parcelas proporcionalmente maiores da agenda de investimento das organizações em geral. Para organizações que buscam melhores condições de competição, o comprometimento de recursos em TI acarreta menor disponibilidade para investimentos em outros recursos potencialmente relevantes, especialmente se ainda não adquiriram economias de escala em tecnologia em condição suficiente nas suas operações (Sanchez \& Albertin, 2009; Zhu, 2004). Com base nesse raciocínio, maiores níveis de investimento em TI levariam à maior opção pela adoção de computação em nuvem, especialmente para aquelas organizações que não estão no estágio de exercer economias de escala de tecnologia em padrão adequado. Dessa forma, afirmamos:

H4: Incrementos no investimento em TI, por parte das organizações, estão positivamente associados a incrementos na possibilidade de adoção da computação em nuvem. 


\section{Modelo e Operacionalização das Variáveis}

Esquematicamente, representamos o modelo de pesquisa conforme a Figura 1.

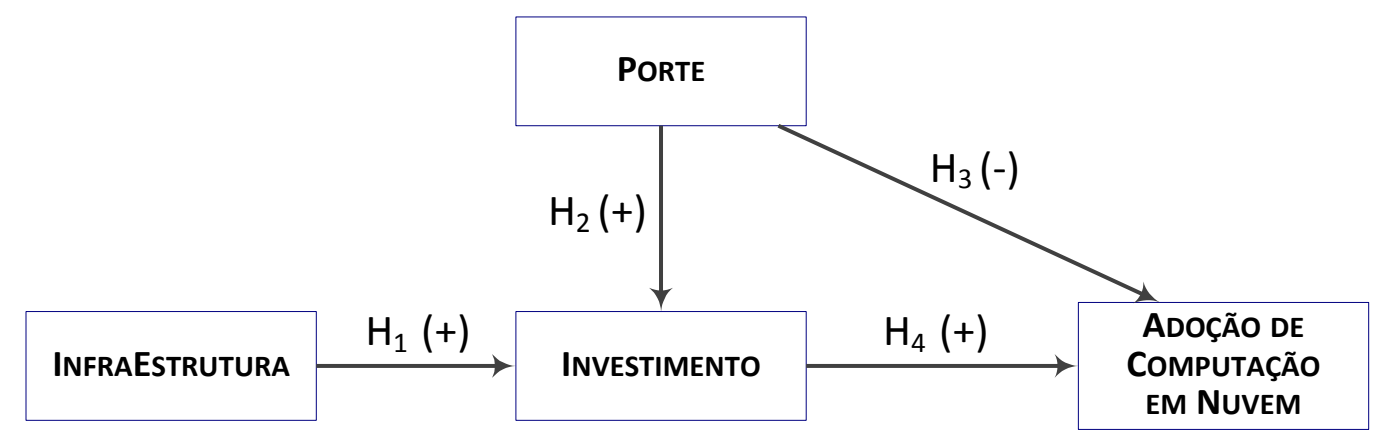

Figura 1. Modelo de Pesquisa e Hipóteses.

A operacionalização dos construtos, nesta pesquisa, dá-se da seguinte forma:

Infraestrutura: esta variável latente constitui-se em um construto reflexivo, que considera o número de servidores na organização, o número de desktops, o número de notebooks e o número de profissionais de TI, incluindo os terceirizados que atuam na organização. Essas variáveis foram consideradas proxies de toda a infraestrutura de TI. Em função da forte assimetria na distribuição de frequências do número de desktops e do número de funcionários de TI, optamos por transformar essa variável por meio da função logaritmo natural. Transformações de variáveis são uma forma de corrigir violações das suposições estatísticas ou melhorar a correlação entre as variáveis (Hair, Anderson, Tatham, \& Black, 2005). Esse recurso é comumente aceito nas pesquisas em que se objetiva reduzir a margem de erro das conclusões que derivam de pressupostos de normalidade dos dados da população, em vista das fortes evidências em contrário na amostra (Gujarati, 2006).

Investimento: essa variável foi operacionalizada por meio de dois indicadores organizados de forma reflexiva, que consistem da informação dos respondentes a respeito dos investimentos efetivamente efetuados no ano de 2009 e da previsão de investimentos a serem efetuados em 2010. Ambas as informações foram fornecidas em 2009, razão pela qual inicialmente consideramos a possibilidade de que apresentariam forte correlação entre si, ou seja, entendemos que poucas organizações apresentariam fortes variações de investimento entre os dois anos, fato posteriormente comprovado por meio da análise do coeficiente Pearson de correlação entre as variáveis. Ambos indicadores foram transformados por meio de seus logaritmos naturais.

Porte: essa variável consiste do logaritmo natural do faturamento do ano de 2009. Vários trabalhos anteriores adotaram o uso do faturamento como proxy de tamanho da empresa (Mitra, 2005; Oh \& Pinsonneault, 2007). Tradicionalmente, entretanto, a distribuição de frequências dessa variável apresenta-se com forte concentração em empresas de menor faturamento, enquanto que algumas empresas apresentam faturamento muito diferente do grupo. Como este trabalho aplica técnicas paramétricas de análise, a normalidade dos dados na população costuma ser importante pressuposto. Tendo em vista que os dados amostrais não apresentaram evidências suficientes de normalidade dessa variável, operacionalizamos a variável Porte como o logaritmo natural do faturamento das empresas constantes na amostra.

Adoção de computação em nuvem: essa variável consiste da resposta direta dicotômica por parte do respondente sobre ter ou não ter a iniciativa de computação em nuvem em andamento em sua organização. Embora haja vários tipos de computação em nuvem, este estudo conta apenas com a informação sobre exercer ou não essa atividade. 


\section{Base de Dados e Resultados}

A base de dados utilizada consiste da pesquisa publicada pela revista INFO EXAME, intitulada As 100 Empresas Mais Ligadas do Brasil e publicada na edição de junho de 2010 (Arima, 2010). Os respondentes consistem de empresas brasileiras que foram solicitadas a responder um questionário eletrônico disponibilizado no início do ano de 2010. As informações foram prestadas pelo principal executivo de TI, pela principal executiva da empresa ou por um representante qualificado. A base completa consta com 100 empresas respondentes, de variados setores e portes.

A partir da análise inicial dos dados, foram identificadas e extraídas da base, por serem considerados outliers, 5 empresas que apresentaram um índice $\mathrm{G}$ calculado como a relação entre investimentos e faturamento de 2009 em valores superiores a 10\%. Esse indicador coloca a empresa em uma situação atípica de acordo com pesquisas sobre a intensidade histórica de investimentos em empresas (Meirelles, 2011). Consideramos que essas empresas fazem parte de um grupo específico e que não é o foco desta pesquisa.

Em função da natureza do estudo, optamos por excluir também as empresas pertencentes ao primeiro quartil de faturamento. A razão dessa escolha decorreu de análise exploratória, que revelou haver forte assimetria nessa distribuição de portes de empresas na amostra, levando ao entendimento de que esse porte desproporcional desse subgrupo de empresas em relação ao restante da amostra não contribuiria para um entendimento das empresas brasileiras em geral, visto que consistem de empresas únicas cada qual em seu setor. Para ilustrar essa condição, ressaltamos que o limite do primeiro quartil em faturamento, pelo qual selecionamos as empresas remanescentes na análise, situou-se em $\mathrm{R} \$ 5,5$ bilhões, enquanto a primeira colocada da amostra geral apresentou faturamento maior que 30 vezes esse limite. A partir dessa escolha, 19 casos foram identificados na base, sendo 4 empresas pertencentes ao setor de energia, 2 mineradoras, 2 distribuidoras de combustíveis, 1 de construção pesada, 6 do setor bancário e securitário, 2 indústrias de bens de consumo, 1 de varejo e 1 de comunicação. Ainda procurando analisar a robustez do modelo resultante, análises adicionais foram implementadas, incluindo esse subgrupo na amostra. Entretanto, seus resultados indicaram que os coeficientes do modelo de análise tornam-se não significantes. A favor da análise, em razão da grande disparidade de porte mencionada acima, do número reduzido de casos por setor e do caráter diferencial que tais empresas apresentam em seus próprios setores, consideramos adequado focar este estudo no grupo remanescente, excluindo, portanto, as 19 observações. Entendemos que estudos futuros poderão contemplar a amostragem específica requerida para que seja possível analisar os setores e portes indicados no subgrupo de grandes corporações extraído.

Do grupo remanescente, foram ainda extraídos mais 20 casos, que apresentaram missing values, resultando 56 casos dos 100 originais. Esse número foi considerado adequado em função de a técnica adotada ser a modelagem por Partial Least Squares, que permite análises de forma adequada considerando o critério mínimo de 10 observações por variável independente, tomadas no construto com maior número de variáveis independentes. Neste estudo, duas variáveis latentes foram modeladas com duas variáveis antecedentes, INVESTIMENTO e ADOÇÃO DE COMPUTAÇÂO EM NUVEM. Em ambos os casos, o número mínimo de observações seria 20, indicando a adequação do número de observações para análise do modelo por essa técnica (Hair et al., 2005).

Os dados descritivos das variáveis antes e após as transformações são apresentados na Tabela 1. 
Tabela 1

Estatística Descritiva das Variáveis

\begin{tabular}{|c|c|c|c|c|c|c|c|c|c|}
\hline & Média & Mediana & $\begin{array}{r}\text { Desvio } \\
\text { Padrão }\end{array}$ & Assimetria & Curtose & Minimo & $\begin{array}{r}\text { Percentil } \\
25\end{array}$ & $\begin{array}{r}\text { Percentil } \\
75\end{array}$ & Máximo \\
\hline Serv & 146 & 99 & 142 & 1,79 & 3,04 & 4 & 49 & 198 & 610 \\
\hline Note & 310 & 185 & 352 & 2,03 & 4,41 & 5 & 80 & 433 & 1.700 \\
\hline Desk & 1.553 & 1.000 & 1.711 & 2,17 & 5,47 & 50 & 407 & 2.000 & 8.700 \\
\hline LnDesk & 6,79 & 6,91 & 1,15 & $-0,37$ & $-0,07$ & 3,91 & 6,01 & 7,60 & 9,07 \\
\hline ProfTI 2009 & 126 & 74 & 182 & 2,92 & 8,26 & 9 & 29 & 125 & 836 \\
\hline LnProfTI 2009 & 4,23 & 4,30 & 1,06 & 0,39 & 0,07 & 2,20 & 3,35 & 4,83 & 6,73 \\
\hline Fat 2009 & 1.479 .578 & 1.045 .622 & 1.388 .856 & 1,08 & 0,40 & 7.572 & 296.250 & 2.525 .000 & 5.110 .596 \\
\hline LnFat 2009 & 13,53 & 13,86 & 1,47 & $-1,07$ & 0,89 & 8,93 & 12,60 & 14,74 & 15,45 \\
\hline InvTI 2009 & 13.995 & 5,610 & 26.293 & 3,28 & 10,84 & 420 & 2.081 & 11.625 & 136.000 \\
\hline LnInvTI 2009 & 8,60 & 8,63 & 1,33 & 0,34 & 0,14 & 6,04 & 7,64 & 9,36 & 11,82 \\
\hline InvTI 2010 & 23.626 & 7.300 & 44.999 & 2,87 & 7,57 & 630 & 3.075 & 19.095 & 200.000 \\
\hline LnInvTI 2010 & 9,01 & 8,90 & 1,39 & 0,52 & 0,07 & 6,45 & 8,03 & 9,86 & 12,21 \\
\hline Nuv & 0,52 & - & 0,50 & $-0,07$ & $-2,07$ & - & - & - & - \\
\hline
\end{tabular}

Nota. $\mathrm{N}=56$

Com o objetivo de analisar se as variáveis PORTE e INVESTIMENTO poderiam ser compostas por indicadores que poderiam ser preditores univariados estatisticamente significantes da adoção da computação em nuvem, inicialmente, foi conduzida uma análise exploratória com esses indicadores sem transformação e transformados, procurando por evidências que pudessem suportar a diferença univariada entre os dois grupos. O agrupamento foi efetuando pela variável NUVEM (Nuv). Os resultados são apresentados na Tabela 2.

Tabela 2

Análise Comparativa entre Grupos

\begin{tabular}{lrrrr} 
& Diferença de médias & Erro Padrão & t & p-valor $^{(\mathbf{p})}$ \\
\hline Fat2009 & -77.913 & 371.771 & $-0,21$ & 0,83 \\
InvTI2009 $^{(\mathbf{L})}$ & -10.062 & 6.823 & $-1,47$ & 0,15 \\
InvTI2010 & -9.690 & 12.017 & $-0,81$ & 0,42 \\
LnFat2009 & 0,30 & 0,39 & 0,77 & 0,45 \\
LnInvTI2009 & $-0,48$ & 0,35 & $-1,37$ & 0,18 \\
LnInvTI2010 & $-0,50$ & 0,37 & $-1,35$ & 0,18 \\
\hline
\end{tabular}

Nota. Nuv coficada como' 0 '. não implementou; ' 1 ' implementou.

${ }^{(L)}$ Teste de Levene é significante ao nível de 0,05 (bicaudal); demais, não significantes; ${ }^{\left({ }^{p}\right)}$ bicaudal.

Como se pode observar desses resultados, nenhum dos indicadores, com ou sem transformação, apresenta diferenças entre grupos estatisticamente significantes. Esse resultado não indica, por si só, que não haja diferenças entre os grupos, mas apenas que estas diferenças não são estatisticamente perceptíveis na condição da amostra levantada. Igualmente, não há implicações em relação à sua utilidade na construção de modelos multivariados, razão pela qual prosseguimos com as análises. 
Procurando estimar a utilidade de aplicar as variáveis em modelos multivariados, procedeu-se à análise inicial de correlações Pearson. As magnitudes das correlações entre as variáveis usadas no modelo e seus níveis de significância são apresentadas na Tabela 3.

Tabela 3

Correlações Pearson entre as Variáveis Usadas no Modelo

\begin{tabular}{llllrrrr} 
& \multicolumn{1}{c}{ Serv } & Note & LnDesk & LnProfTI & LnFat2009 & $\begin{array}{r}\text { LnInvTI } \\
\mathbf{2 0 0 9}\end{array}$ & $\begin{array}{r}\text { LnInvTI } \\
\mathbf{2 0 1 0}\end{array}$ \\
\hline Note & $0,289^{*}$ & & & & & & \\
LnDesk & $0,623^{* *}$ & $0,471^{* *}$ & & & & & \\
LnProfTI 2009 & $0,719^{* *}$ & $0,312^{*}$ & $0,511^{* *}$ & & & & \\
LnFat 2009 & 0,24 & $0,518^{* *}$ & $0,666^{* *}$ & 0,20 & & & \\
LnInvTI 2009 & $0,487^{* *}$ & $0,491^{* *}$ & $0,563^{* *}$ & $0,494^{* *}$ & $0,615^{* *}$ & & \\
LnInvTI 2010 & $0,554^{* *}$ & $0,489^{* *}$ & $0,564^{* *}$ & $0,607^{* *}$ & $0,554^{* *}$ & $0,899^{* *}$ & \\
Nuv & 0,155 & 0,012 & 0,024 & 0,183 & $-0,103$ & 0,181 & 0,181 \\
\hline
\end{tabular}

Nota. $\mathrm{N}=56$.

* Correlação é significante ao nível de 0,05 (bicaudal); ** Correlação é significante ao nível de 0,01 (bicaudal).

O modelo da Figura 1 foi então analisado por meio de equações estruturais, usando a técnica de Partial Least Squares (PLS). Inicialmente, procedendo à análise do modelo de medidas, os resultados indicam que a validade convergente é adequada, tendo em vista o critério de que a Variância Média Extraída - AVE - de cada variável latente deve ser superior a 0,5. De acordo com a tabela 4, observamos que as duas variáveis latentes (INFRA e INVEST) apresentaram valores superiores ao limite, significando que não foram deixados de lado aspectos relevantes dos indicadores que compõem os construtos.

Em relação à consistência interna, dois critérios foram utilizados: a análise dos valores do Alpha de Cronbah e da Confiabilidade Composta. Tradicionalmente, os estudos com construtos reflexivos (Bagozzi, 2011) consideram o Alpha de Cronbach com a recomendação de que deva apresentar minimamente valores acima de 0,7 , o que é frequente em estágios iniciais de pesquisas (Nunnally, 1978) e acima de 0,80 em pesquisas maduras (Clark \& Watson, 1995). No caso dos dados da Tabela 4 , podemos observar que os valores situam-se acima desse nível. Entretanto, o Alpha de Cronbach é calculado supondo pesos iguais para os diversos indicadores de cada variável latente, o que foi uma estratégia muito comum em pesquisas, no passado, quando se compunha os escores das variáveis latentes por meio da média simples de seus indicadores (Jarvis, Mackenzie, \& Podsakoff, 2003). Atualmente, a técnica de PLS permite compor a variância do indicador de forma a maximizar o poder de explicação do modelo. Isso é feito pela criação de uma proxy de cada variável latente, por meio de sucessivas aproximações com algoritmos de modelos lineares que minimizam de maneira local o quadrado dos erros, o que maximiza o coeficiente de determinação de cada variável latente (Dijkstra, 2010). Isso, usualmente, resulta em pesos divergentes entre os indicadores na composição dos escores da variável latente. Assim, usamos a Confiabilidade Composta para essa análise, que é calculada sem a predefinição de pesos iguais para os indicadores (Jarvis et al., 2003). De acordo com a Tabela 4, os valores estão adequados. 
Tabela 4

Variância Média Extraída (AVE) e Confiabilidade

\begin{tabular}{lrrr} 
& AVE & Confiabilidade Composta & Alpha de Cronbach \\
\hline INFRA & 0,622 & 0,867 & 0,792 \\
INVEST & 0,949 & 0,974 & 0,947 \\
PORTE & 1,00 & 1,00 & 1,00 \\
NUVEM & 1,00 & 1,00 & 1,00 \\
\hline
\end{tabular}

Em relação à validade discriminante, a Tabela 5 compara a raiz quadrada da variância extraída (AVE) com as intercorrelações entre as demais variáveis latentes. A raiz de AVE é uma medida de quanto os indicadores em um construto reflexivo são adequados a representar a variância da variável latente que representam. Pelo critério de Fornell e Larcker (1981), a raiz de AVE deve ser maior que as intercorrelações entre as demais variáveis latentes, que é a correlação parcial entre os construtos, 2 a 2, ou seja, a correlação entre dois construtos, excluídas as influências entre os demais construtos. Observando-se a Tabela 5, vemos que todos os valores da raiz de AVE, posicionados nas diagonais, são maiores que os valores das colunas e linhas respectivas, indicando que o modelo de medidas apresenta adequada validade discriminante entre construtos. Por exemplo, as intercorrelações entre os construtos INFRA-INVEST $(0,69)$, INFRA-NUVEM $(0,12)$ e INFRA-PORTE $(0,52)$ devem apresentar valores menores que a diagonal INFRA-INFRA $(0,79)$. Isso revela que os indicadores de INFRA são mais adequados para medir esse construto do que os demais construtos (INVEST, NUVEM e PORTE), o que atesta a validade discriminante dessa operacionalização. De forma similar, INVEST-INVEST $(0,97)$ possui valor superior a INFRA-INVEST $(0,69)$, INVEST-NUVEM $(0,19)$ e INVEST-PORTE $(0,60)$. Igualmente, NUVEM-NUVEM $(1,0)$ é superior a INFRA-NUVEM $(0,12)$, INVEST-NUVEM $(0,19)$ e NUVEM-PORTE $(-0,10)$. Por fim, PORTE-PORTE $(1,0)$ apresenta-se superior a INFRA-PORTE $(0,52)$, INVEST-PORTE $(0,60)$ e NUVEM-PORTE $(-0,10)$.

Tabela 5

Correlações e Variância Média Extraída, por construto ${ }^{(1)}$

\begin{tabular}{lrrrr}
\cline { 2 - 5 } & INFRA & INVEST & NUVEM & PORTE \\
\hline INFRA & $\mathbf{0 , 7 9}$ & & & \\
INVEST & 0,69 & $\mathbf{0 , 9 7}$ & & \\
NUVEM & 0,12 & 0,19 & $\mathbf{1 , 0 0}$ & \\
PORTE & 0,52 & 0,60 & $-0,10$ & $\mathbf{1 , 0 0}$ \\
\hline
\end{tabular}

Nota. ${ }^{(1)}$ A raiz quadrada da Variância Média Extraída é representada na diagonal.

Ainda em relação à validade discriminante, a Tabela 6 apresenta os escores baseados em Análise Fatorial Confirmatória, cuja análise se processa sobre as cargas dos indicadores de uma variável latente, que devem ter valores superiores às cargas desses mesmos indicadores em variáveis latentes para as quais os indicadores não são destinados. Adicionalmente, a confiabilidade do modelo de medidas é aferida pela análise do valor do escore de cada indicador em cada construto a que se destina, visando a garantir que pelo menos metade da variância do indicador seja apropriada ao construto, assim, levando o escore a ser maior que o valor da raiz quadrada de 0,5 , ou seja, pelo menos 0,71 (Gefen, Straub, \& Boudreau, 2000). No caso presente, apenas o indicador Note não atinge esse patamar. Ainda assim, como o valor é de 0,64, próximo ao limite, e como desejamos representar ao menos uma parcela da variabilidade desse item de Infraestrutura, em lugar de eliminar o indicador, optamos por mantê-lo. 
Tabela 6

Validade Discriminante (Cargas Fatoriais)

\begin{tabular}{lrrrr}
\cline { 2 - 5 } & INFRA & INVEST & NUVEM & PORTE \\
\hline LnDesk & 0,84 & 0,58 & 0,02 & 0,67 \\
LnProfTI2009 & 0,82 & 0,57 & 0,18 & 0,20 \\
Note & 0,64 & 0,50 & 0,01 & 0,52 \\
Serv & 0,84 & 0,53 & 0,15 & 0,24 \\
LnInvTI2009 & 0,65 & 0,97 & 0,18 & 0,61 \\
LnInvTI2010 & 0,71 & 0,97 & 0,18 & 0,55 \\
Nuv & 0,12 & 0,19 & 1,00 & $-0,10$ \\
LnFat2009 & 0,52 & 0,60 & $-0,10$ & 1,00 \\
\hline
\end{tabular}

A análise da qualidade do modelo estrutural dá-se pela constatação da significância das cargas de caminhos do modelo, conforme a Tabela 7, cuja estimativa foi feita por meio da técnica de subamostragem (bootstraping) para 1.000 subamostras aleatórias de 56 casos, com reposição (Efron, 1979). Essa técnica permite aferir com adequada precisão modelos complexos de múltiplos relacionamentos e relativamente poucas observações. No caso deste estudo, todos os caminhos de relacionamento do modelo apresentaram significância ao nível mínimo de $5 \%$ ou $0,1 \%$, conforme indicado. Além disso, a técnica envolveu o chamado PLS Generalised Linear Regression, onde o algoritmo PLS atua no esquema de um Probit, dado que se implemente o bootstrap aleatório (Bastien, Vinzi, \& Tenenhaus, 2005), o que é necessário em função da variável dependente ser dicotômica. Assim, os coeficientes de caminhos no modelo que levam à variável NUVEM podem ser diretamente interpretados de maneira similar aos demais coeficientes.

Como resultado final, o poder de explicação do modelo alcançou $56,1 \%$ para investimentos e $10,6 \%$ para computação em nuvem. A magnitude e sinal dos efeitos são consistentes com as hipóteses formuladas a partir da revisão de literatura.

Tabela 7

Coeficientes do Modelo Estrutural e Significâncias ${ }^{(1)(2)}$

\begin{tabular}{lrrrrr} 
& INVEST & NUVEM & $\begin{array}{r}\text { Erro padrão } \\
(\mathbf{S E})\end{array}$ & $\begin{array}{r}\text { Estatística t } \\
(|\mathbf{M} / \mathbf{S E}|)\end{array}$ & $\boldsymbol{p}$ valor \\
\hline INFRA & $0,52 * * *$ & $0,20 * * *(3)$ & 0,093 & 5,648 & 0,000 \\
\hline \multirow{2}{*}{ PORTE } & $0,33 * * *$ & $-0,21 *(3)$ & 0,089 & 3,671 & 0,001 \\
& & $-0,33 *$ & 0,157 & 2,130 & 0,038 \\
\hline INVEST & & $0,39 *$ & 0,156 & 2,483 & 0,016 \\
\hline $\mathbf{R}^{2}$ & $\mathbf{5 6 , 1 \%}$ & $\mathbf{1 0 , 6 \%}$ & & & \\
\hline
\end{tabular}

Nota. ${ }^{(1)} * \mathrm{p}<0.05 ; * * \mathrm{p}<0.01 ; * * * \mathrm{p}<0.001$; (NS) Não significante. ${ }^{(2)}$ Estimativa efetuada por técnica de Bootstrap de 1000 subamostras, com reposição; ${ }^{(3)}$ Magnitude dos efeitos mediados por Investimento. 


\section{Discussão}

Os dados revelam que a infraestrutura está positiva e significantemente associada aos investimentos de TI $(0,52 ; \mathrm{p}<0,001)$, o que representa evidência a favor do suporte à primeira hipótese. Concorrem para que as organizações com mais infraestrutura procurem ampliar seus investimentos em TI aspectos como as necessidades de expansão de negócio, a busca por economias de escala e até mesmo a obsolescência da tecnologia de infraestrutura, conquanto esta ocorra em velocidade menor que a dos aplicativos (Brynjolfsson \& Hitt, 2000). Nesta pesquisa, a operacionalização da variável latente infraestrutura incluiu indicadores como desktops, notebooks, servidores e pessoal de TI. Embora caracterizados como infraestrutura, todos esses indicadores são também afetados por iniciativas no campo das aplicações, investimentos das organizações que mais diretamente respondem às condições de competitividade e crescimento, mas que se refletem como expansão em infraestrutura. Neste sentido, é possível que organizações com mais infraestrutura possam alcançar economias de escala computacional suficientes para que possam se lançar na implementação de aplicações às quais empresas com menor nível de infraestrutura não tenham acesso (Sanchez \& Albertin, 2009). Já o nível de significância de $0,1 \%$ consiste de forte evidência a favor do entendimento de que as organizações que possuem mais infraestrutura investem mais em tecnologia da informação.

O porte da empresa, por sua vez, apresenta associação positiva e significante com o investimento em TI $(0,33 ; p<0,001)$, o que igualmente suporta a hipótese aventada (H2), em função da articulação da revisão de literatura. Dois grandes fatores parecem contribuir para esse efeito: os crescentes custos de coordenação advindos dos incrementos de complexidade dos processos e de estrutura a que estão expostas as organizações maiores (Sanchez \& Albertin, 2009) e a busca por ganhos de escala que podem reforçar sua posição competitiva de forma a proteger a organização das ações de competidores menores, e mais especializados em segmentos de mercado ou atividades (Damanpour, 2010). Adicionalmente, o risco da falta de infraestrutura em organizações maiores pode ser percebido como maior pela limitação na capacidade de fornecimento dos fornecedores. Assim, às grandes organizações, sua economia de escala permite considerar a aquisição de capacidade de infraestrutura sempre que fornecedores apresentarem capacidades limitadas ou comprometidas (Duncan, 1995; Renkema, 1998).

Já a aquisição de computação em nuvem está negativamente relacionada com o porte da organização $(-0,33 ; \mathrm{p}<0,05)$, o que apresenta evidências a favor da hipótese (H3), levantada com base na argumentação teórica. Organizações de maior porte tendem a estar em condições de produzir economias de escala que se tornam a base de sua vantagem competitiva. Isso se dá não apenas no sentido da maior demanda que podem atender, mas também na capacidade de aquisição de recursos e competências diferenciais (Barua et al., 1991). Dessa maneira, organizações de menor porte, usualmente em situação de adquirir posição competitiva, tendem a adotar a computação em nuvem mais que organizações de maior porte como forma de liberar recursos para adquirir capacidade competitiva, enquanto organizações de maior porte buscam proteger seus ativos competitivos, portanto evitando exteriorizá-los, uma vez que possuem capacidade de investimento para tanto (Gomes et al., 2009).

Por fim, os resultados indicam que os investimentos em TI estão positivamente associados à adoção da computação em nuvem $(0,39 ; \mathrm{p}<0,05)$. Os recursos de TI são caros e a demanda por recursos nessa área representa uma pressão que confronta todas as organizações (Zhu, 2004). Mesmo as organizações que podem manter seus investimentos consideram a possibilidade de adotar algumas iniciativas de computação em nuvem para tornar-se mais líquidas e aumentar sua capacidade de investimento em projetos alternativos (Sanchez \& Albertin, 2009). Esta conclusão, aparentemente contraditória em relação à anterior, é harmonizada pela argumentação de que organizações de maior porte também possam dedicar-se a iniciativas de computação em nuvem, mas que o fazem em menor intensidade relativa do que empresas de menor porte.

Em resumo, temos que a pesquisa apresentou evidências suficientes para suportar as quatro hipóteses propostas. 


\section{Conclusão e Limitações}

Com base em dados secundários, esta pesquisa levantou evidências a respeito dos fatores antecedentes da adoção pelas organizações da computação em nuvem. Por ser um fenômeno recente, assim como vários outros momentos em que novas tecnologias e modelos de negócio chegam ao mercado, a computação em nuvem vem sendo apresentada como uma solução quase inquestionável para as restrições de recursos e capacidades das organizações.

É importante poder analisar mais detidamente seus condicionantes e resultados para que os gestores possam tomar melhores decisões quanto a um dos recursos mais caros e estratégicos para as organizações, como a tecnologia da informação. Por exemplo, organizações de maior porte tendem a apresentar economias de escala que as permitirão escolher qual estratégia de computação em nuvem seguir. Em geral, organizações de maior porte podem contar com economias de escala internas que as habilitem à decisão de não optarem pela computação em nuvem. Essa decisão adquire importância estratégica adicional porque a contratação de um fornecedor em nuvem pode habilitá-lo técnica e economicamente a obter e repassar uma economia de escala a potenciais concorrentes menores. Se mantida internamente, a capacidade computacional e a competência técnica podem transformar-se em uma barreira à operação eficiente de competidores que não contam com essa competência.

Da perspectiva gerencial, as conclusões a que chegamos permitem indicar que ações estratégicas às organizações podem implementar suas potenciais consequências. Do ponto de vista da gestão de fornecedores nesse segmento, alguns dos elementos que foram identificados permitem que sejam ofertadas soluções de valor mais adequadas às demandas das organizações clientes. Por exemplo, fornecedores podem traçar estratégias para atuar em segmentos de organizações com menor quantidade de infraestrutura, cujo massivo investimento pode representar uma barreira ao crescimento dessas organizações. Adicionalmente, organizações com grandes volumes de infraestrutura podem ver-se interessadas em contratar serviços de computação em nuvem à medida que seja previsível que seus ativos tecnológicos estejam ameaçados pela obsolescência, ou o custo de manter seu parque tecnológico atualizado esteja drenando recursos substantivos para novos movimentos estratégicos.

Em suma, a adoção da computação em nuvem não pode ser tomada como uma consequência inevitável da disponibilidade advinda da evolução tecnológica, mas como resultado de movimentos estratégicos que organizações, clientes, fornecedoras e competidores planejem executar.

Como limitações deste estudo, podemos listar o fato de que a base de dados não se constituiu em uma amostra aleatória e que, por essa razão, as generalizações para todas as empresas devem ser feitas com devida ressalva. Do ponto de vista da operacionalização, o fato de ter sido fundamentado em dados secundários, inibe a possibilidade de construir variáveis latentes mais robustas, por limitação de indicadores. Futuras pesquisas podem tratar desse aspecto ao incluir novos indicadores para melhor representação dos construtos medidos.

Outra limitação refere-se ao recorte da amostra, que focou os 3 quartis de menor faturamento na base (abaixo de $\mathrm{R} \$ 5,5$ bilhões/ano), como forma de homogeneizar o grupo e permitir a produção de interpretações mais robustas. O grupo remanescente, entretanto, desperta a curiosidade de investigação para que se possam caracterizar as condições de investimento e adoção de computação em nuvem naquelas condições.

Como indicações de pesquisas futuras, recomenda-se que dados primários sejam coletados e que a variável nuvem possa ser operacionalizada de maneira complexa em dois níveis, com base nas classificações aqui apresentadas para esse tipo de atividade. Adicionalmente, a observação de dados longitudinais pode permitir melhor compreensão do fenômeno de adoção de computação em nuvem, tendo em vista tratar-se de uma oferta relativamente recente e um fenômeno ainda em andamento.

Artigo recebido em 20.06.2011. Aprovado em 28.07.2012. 


\section{Nota}

\footnotetext{
${ }^{1}$ Uma versão preliminar deste artigo foi apresentada no III Encontro de Administração da Informação (EnADI). Porto Alegre: Anpad - Associação Nacional dos Programas de Pós-Graduação em Administração, 2011.
}

\section{Referências}

Arima, K. (2010, junho). Tecnologia = Lucros. Revista Info-Exame, pp. 65-71. Recuperado de http://info.abril.com.br/arquivo/2010/jun.shtml

Armbrust, M., Fox, A., Griffith, R., Joseph, A. D., Katz, R., Konwinski, A., Lee, G., Patterson, D., Rabkin, A., Stoica, I., \& Zaharia, M. (2010). A view of cloud computing. Communications of the ACM, 53(4), 50-58. doi:10.1145/1721654.1721672

Armstrong, C. P., \& Sambamurthy, V. (1999). Information technology assimilation in firms: the influence of senior leadership and IT infrastructures. Information Systems Research, 10(4), 304327. doi:10.1287/isre.10.4.304

Bagozzi, R. P. (2011). Measurement and meaning in information systems and organizational research: methodological and philosophical foundations. MIS Quarterly, 35(2), 261-292.

Bannister, F., \& Remenyi, D. (2000). Acts of faith: instinct, value and IT investment decisions. Journal of Information Technology, 15(3), 231-241. doi:10.1080/02683960050153183

Barua, A., Kriebel, C. H., \& Mukhopadhyay, T. (1991). An economic analysis of strategic information technology investments. MIS Quarterly, 15(3), 313-331. doi:10.2307/249643

Bastien, P., Vinzi, V. E., \& Tenenhaus, M. (2005). PLS generalised linear regression. Computational Statistics \& Data Analysis, 48(1), 17-46. doi: 10.1016/j.csda.2004.02.005

Brynjolfsson, E., \& Hitt, L. M. (2000). Beyond computation: information technology, organizational transformation and business performance. The Journal of Economic Perspectives, 14(4), 23-48. doi:10.1257/jep.14.4.23

Bublitz, E. (2010). Catching the cloud: managing risk when utilizing cloud computing. Property \& Casualty Risk \& Benefits Management, 114(39), 12-16.

Byrd, T. A., \& Turner, D. E. (2000). Measuring the flexibility of information technology infrastructure: exploratory analysis of a construct. Journal of Management Information Systems, $17(1), 167-208$.

Byrd, T. A., Pitts, J. P., Adrian, A. M., \& Davidson, N. W. (2008). Examination of a path model relating information technology infrastructure with firm performance. Journal of Business Logistics, 29(2), 161-187. doi: 10.1002/j.2158-1592.2008.tb00091.x

Clark, L. A., \& Watson, D. (1995). Constructing validity: basic issues in objective scale development. Psychological Assessment, 7(3), 309-319. doi: 10.1037/1040-3590.7.3.309

Clemons, E. K., \& Weber, B. W. (1990). Strategic information technology investments: guidelines for decision making. Journal of Management Information Systems, 7(2), 9-28.

Damanpour, F. (2010). An integration of research findings of effects of firm size and market competition on product and process innovations. British Journal of Management, 21(4), 9961010. doi: $10.1111 / \mathrm{j} .1467-8551.2009 .00628 . \mathrm{x}$ 
Davern, M. J., \& Kauffman, R. J. (2000). Discovering potential and realizing value from information technology investments. Journal of Management Information Systems, 16(4), 121-143.

Dijkstra, T. K. (2010). Latent variables and indices: Herman's word's basic design and partial least squares. In V. E. Vinzi, W.W. Chin, J. Henseler, \& H. Wang (Eds.), Handbook of partial least squares: concepts, methods and applications (pp. 23-46). New York: Springer.

Duncan, N. B. (1995). Capturing flexibility of information technology infrastructure: a study of resource characteristics and their measure. Journal of Management Information Systems, 12(2), $37-57$.

Durkee, D. (2010). Why cloud computing will never be free. Communications of the ACM, 53(5), 6269. doi: 10.1145/1735223.1735242

Efron, B. (1979). Bootstrap methods: another look at the jackknife. Annals of Statistics, 7(1), 1-26. doi: $10.1214 / \mathrm{aos} / 1176344552$

Fano, A., \& Gershman, A. (2002). The future of business services in the age of ubiquitous computing. Communications of the ACM, 45(12), 83-87. doi:10.1145/585597.585620

Ford, S. (2010). Managing your global business with cloud technology. Financial Executive, 26(8), 56-59.

Fornell, C., \& Larcker, D. F. (1981). Evaluating structural equation models with unobservable variables and measurement error. Journal of Marketing Research, 18(1), 39-50.

Gefen, D., Straub, D. W., \& Boudreau, M. C. (2000). Structural equation modeling and regression: guidelines for research practice. Communications of the Association of Information Systems, 4(7), 1-77.

Gomes, C. M., Kruglianskas, I., \& Scherer, F. L. (2009). Company size effect in innovative performance. Journal of Technology Management \& Innovation, 4(4), 13-31. doi: $10.4067 / \mathrm{S} 0718-27242009000400002$

Gujarati, D. (2006). Econometria básica. Rio de Janeiro: Elsevier.

Hair, J. F., Jr., Anderson, R. E., Tatham, R. L. \& Black, W. C. (2005) Análise multivariada de dados (5a ed.). Porto Alegre: Bookman.

Holm, C., \& Rikhardsson, P. (2008). Experienced and novice investors: does environmental information influence investment allocation decisions? European Accounting Review, 17(3), 537-557. doi:10.1080/09638180802016627

Iyer, B., \& Henderson, J.C. (2010). Preparing for the future: understanding the seven capabilities cloud computing. MIS Quarterly Executive, 9(2), 117-131.

Jarvis, C. B., Mackenzie, S. B., \& Podsakoff, P. M. (2003). A critical review of construct indicators and measurement model misspecification in marketing and consumer research. Journal of Consumer Research, 30(2), 199-218. doi: 10.1086/376806

Katzan, H., Jr. (2010). On an ontological view of cloud computing. Journal of Service Science, 3(1), $1-6$.

McAfee, A. P. (2006). Mastering the three worlds of information technology. Harvard Business Review, 84(11), 141-149.

Meirelles, F. S. (2011). Administração de recursos de tecnologia da informação (Relatório da 22a pesquisa anual). São Paulo, SP, Fundação Getúlio Vargas. 
Mitra, S. (2005). Information technology as an enabler of growth in firms:an empirical assessment. Journal of Management Information Systems, 22(2), 279-300.

Nunnally, J. (1978). Psychometric theory. New York: McGraw-Hill.

Oh, W., \& Pinsonneault, A. (2007). On the assessment of the strategic value of information technologies: conceptual and analytical approaches. MIS Quarterly, 31(2), 239-265.

Renkema, T. J. W. (1998). The four P's revisited: business value assessment of the infrastructure impact of IT investments. Journal of Information Technology, 13(3), 181-190. doi: $10.1080 / 026839698344828$

Ryan, S. D., \& Harrison, D. A. (2000). Considering social subsystem costs and benefits in information technology investment decisions: a view from the field on anticipated payoffs. Journal of Management Information Systems, 16(4), 11-40.

Ryan, W. M., \& Loeffler, C. M. (2010). Insights into cloud computing. Intellectual. Property \& Technology Law Journal, 22(11), 22-28.

Sanchez, O. P., \& Albertin, A. L. (2008). Seleção de alternativas de outsourcing. In A. L. Albertin \& O. P. Sanchez (Orgs.), Outsourcing de TI: impactos, dilemas, discussões e casos reais (pp. 2745). Rio de Janeiro: FGV.

Sanchez, O. P. \& Albertin, A. L. (2009). A racionalidade limitada das decisões de investimento em tecnologia da informação. Revista de Administração de Empresas, 49(1), 86-106. doi: 10.1590/S0034-75902009000100010

Santos, B. L. dos (1991). Justifying investments in new information technologies. Journal of Management Information Systems, 7(4), 71-89.

Simon, H. A. (1990). Information technologies and organizations. The Accounting Review, 65(3), 658667.

Weill, P., \& Broadbent, M. (1998). Leveraging the new infrastructure: how market leaders capitalize on information technology. Boston, MA: Harvard Business School Press

Zhu, K. (2004). The complementarity of information technology infrastructure and e-commerce capability: a resource-based assessment of their business value. Journal of Management Information Systems, 21(1), 167-202. 